\title{
Fast Energy Exchange between Fixed Supercapacitor and On-board Supercapacitor
}

\author{
${ }^{\text {"1 } M a n j u s h a ~ P a l a n d u r k a r, ~}{ }^{2}$ Manisha Gaikwad, ${ }^{3}$ Mohan Renge \\ ${ }^{1,2,3}$ Shri Ramdeobaba College of Engineering \& Management, Nagpur \\ Email: ${ }^{1}$ palandurkarmv@rknec.edu, ${ }^{2}$ gaikwadmb@rknec.edu, ${ }^{3}$ rengemm@rknec.edu
}

Received: 06th October 2019, Accepted: 20th November 2019, Published: 31st December 2019

\begin{abstract}
Supercapacitor (SC) is an electrical energy storage device that bridges the gap between electrolytic capacitor and rechargeable battery. It can accept and deliver charge much faster than battery as well it has a high power density. This property makes them a good choice to be used in electric vehicles like buses and cars. To recharge the on-board SC module used in electric vehicle in less time, a high amount of power is required. If this power is taken from distribution network, then high magnitude of peak current is absorbed from the supply which can create constraint on power network. To overcome this problem, on-board SC module is charged through fixed SC module which acts as energy buffers through DC- DC converters. In this paper, instead of using boost and buck converter, a double stage conversion technique is proposed for the fast energy exchange between the two energy systems with the help of MATLAB Simulink software and by experimental results.
\end{abstract}

Keywords

Supercapacitor (SC), DC- DC Converter, Digital Signal Controller.

\section{Introduction}

Day by day, the world is moving towards the eco friendly solutions. One of being them is the Electric Vehicles (EV) which is the source of zero carbon emissions. In EV, the batteries are the main energy source which provides anatomy of the vehicle. Battery stores the electrical energy in the form of electro chemical process. The batteries have been used since long time due to their electrical qualities such as higher energy density, voltage stability. The main demerit regarding the batteries is their number of charge/discharge cycles which are limited [1]. The battery life is affected if the number of charge/discharge cycles exceeds its limit. It also falls short in supplying the peak power demands by the load. To overcome the limitations of the battery, another energy storage device available is supercapacitor (SC). Supercapcitor is electrochemical double layer capacitor (ECDL) but no chemical reactions are involved during the energy storage process. Due to this, it can be charged discharged thousands of times [1]. The property of SC to provide a high power exchange makes them stand apart from the battery. Because of this property, supercapacitor module can be used as energy storage system in electric vehicles such as buses and cars. If supercapacitors, as a module, are used as main energy storage system for an electric bus, then the needed stored energy has to power the bus from the start station and to the next refilling station.

The supercapacitor module should be rapidly recharged simultaneously with the exchange of passengers. During the recharging of the supercapacitor tank, the power which is injected in the tank is taken directly from the power network [2]. The power network has to provide high magnitude power pulses in short duration. This high magnitude of power can't be supplied when a distribution network is weak. Hence, the properties of supercapacitors which require fast charging impose high constraints on the supply network that cannot be tolerated. To overcome this problem, on-board supercapacitor module is charged through fixed supercapacitor module which acts as energy buffers [3] [4].

The main objective of this proposed work is to rapidly charge the on-board SC module, when the bus is at charging station. The idea is to take the energy from another fixed SC module, which is placed in the refilling station. Fixed SC module will be used to transfer this energy, taking advantages of the high instantaneous power supercapacitores can provide or store. The high magnitude power pulses are needed for fast loading of the onboard SC module supported by the fixed SC module. During charging process, fixed SC module is disconnected from power network. When the bus leaves the charging station, then fixed SC module is charged by power network, to prepare it for next coming bus. The time allowed for charging of fixed SC module is the time between two buses. Due to increased charging time, the value of charging current required to charge fixed SC module is less for the same amount of energy. This reduces the value of the instantaneous power taken from the power network. 


\section{Materials and Methods}

A.Fixed SC Module

These are the fixed supercapacitors which are charged by power network. In this paper, the fixed SC module is made up of six supercapacitor cell connected in series each having a capacitance of $100 \mathrm{~F}$ and voltage of $2.7 \mathrm{~V}$. The maximum ESR DC and absolute maximum current of $100 \mathrm{~F}$ supercappacitor is $15 \mathrm{~m} \Omega$ and $36 \mathrm{~A}$ respectively. The total voltage of the module is $16.2 \mathrm{~V}$ with the equivalent capacitance of $16.66 \mathrm{~F}$. [5] The total energy stored can be calculated using the formula $E=(1 / 2) * C^{*} V^{2}$

Where, $\mathrm{E}$ is energy stored in joule, $\mathrm{C}$ is total capacitance in farad and $\mathrm{V}$ is total voltage of SC module.

\section{B.On-board SC Module}

These are the mobile supercapacitors which are placed in the vehicle and are charged from the fixed SC module. The on-board SC module consists of eight supercapacitor cells connected in series. Each cell has capacitance of $50 \mathrm{~F}$ and voltage rating of $2.7 \mathrm{~V}$. The maximum ESR DC and absolute maximum current of 50F supercappacitor is $20 \mathrm{~m} \Omega$ and 27 A respectively. The total voltage of string is $21.6 \mathrm{~V}$ with equivalent capacitance of $6.25 \mathrm{~F}$.

\section{DC-DC Converter}

The challenge faced by supercapacitor is that their output voltage is continuously changing during discharging operation. Here, the power electronics converter plays an important role to overcome this problem. Generally, dc-dc converters are preferred to be used with these SC modules. This converter is used to either step up or step down the input DC voltage to a desired value. In this work, a double stage converter shown in Fig. 1 is implemented which interface the fixed SC module and on-board SC module [4] [6]. This converter comprises of two stages i.e. boost converter stage and buck converter stage which are described below.

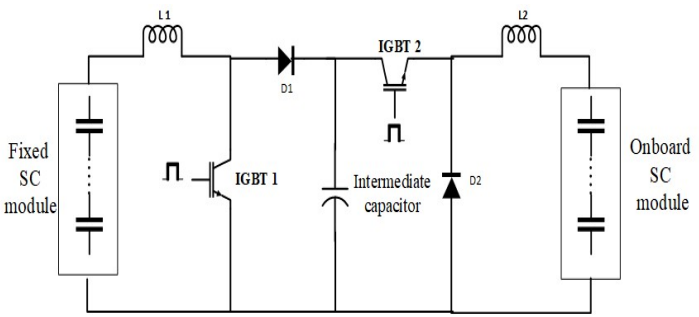

Fig. 1: Double Stage Convertor

In first stage, boost converter is implemented which contributes in elevation of voltage from fixed SC module to intermediate capacitor. The voltage of fixed SC module goes on decreasing as there is transfer of energy to the on-board module. In second stage, buck converter is designed in which the voltage across intermediate capacitor is stepped down to a desired value to charge the onboard SC module.

\section{Control Strategy for Fast Transfer}

The control circuit consists of two loops i.e. current control loop and voltage control loop. Fig. 2(a) shows the topology for the current control operation which is implemented with the help of PI controller. The desired reference value of current is compared with the actual current sensed from the inductor of first stage converter. The error generated is then fed to the PI controller to obtain the switching pulses which are given to the IGBT-1 switch of the first stage converter. The second loop, as shown in Fig. 2(b), also implements a PI controller for maintaining a constant voltage across the intermediate capacitor. A set point value of a desired voltage is considered which is compared with the actual voltage of intermediate capacitor to generate an error. The PI controller then decides the duty cycle which is compared with a repeating sequence to generate the switching pulses for the IGBT-2 switch of second stage converter.

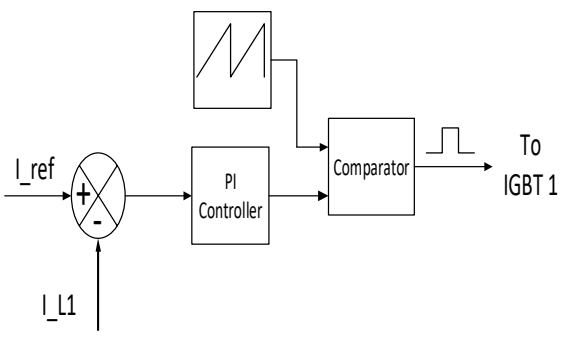

Fig. 2(a): Current Control Loop

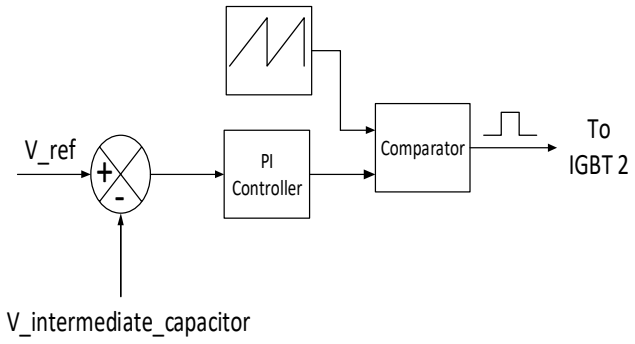

Fig. 2(b): Voltage Control Loop 


\section{SIMULINK Model}

In order to implement the above proposed work, the SIMULINK model is designed by using MATLAB software. As shown in Fig. 3, power circuit includes the double stage converter which interfaces the fixed SC module and on-board SC module. The rating of fixed SC module and On-board SC module are chosen $15 \mathrm{~V}$, $16.66 \mathrm{~F}$ and $20 \mathrm{~V}, 6.25 \mathrm{~F}$ respectively while inductances value for both boost $\mathrm{n}$ buck converter are chosen as $1 \mathrm{mH}$ and intermediate capacitance value is considered as $500 \mu \mathrm{F}$.

The control circuit includes the voltage controller to maintain desired constant voltage across the intermediate capacitor. It also includes the current control loop which controls the current through fixed SC module.

Initially, the fixed SC module is considered as fully charged voltage of $15 \mathrm{~V}$ whereas on-board SC module is considered as half charged up to $10 \mathrm{~V}$. This is half of its maximum voltage rating of $20 \mathrm{~V}$. As the voltage across on-board SC module is half, they are not capable of handling high instantaneous power which will be supplied by the fully charged fixed SC module. Due to this reason, the current provided by the fixed SC module has to be set at a value which must be lower than its absolute maximum limit. Therefore, the current through fixed SC module is set at $10 \mathrm{~A}$.

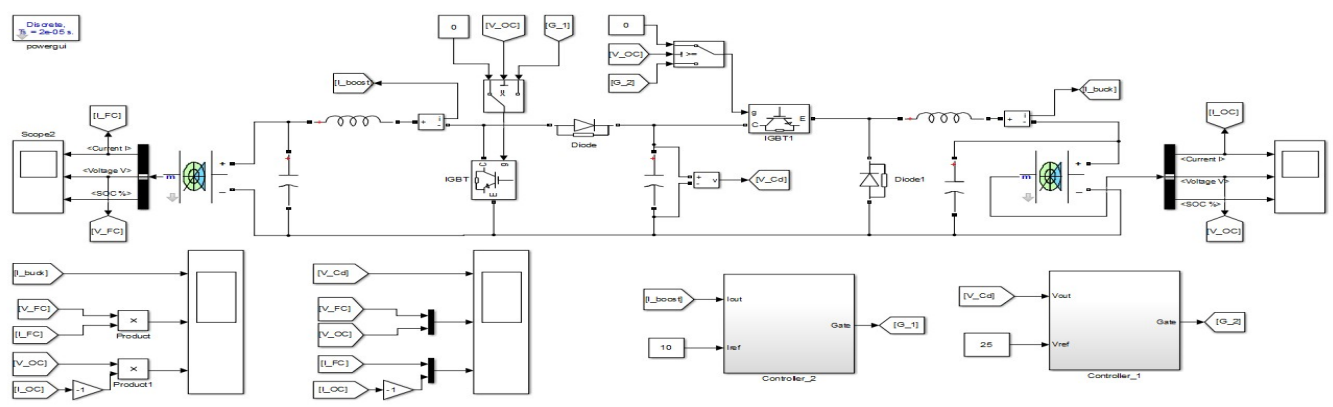

Fig. 3: Simulink Model of Double Stage Conveter

\section{SIMULINK Result}

Fig. 4(a) shows the constant voltage across the intermediate capacitor which is controlled by implementing close loop operation. A voltage control closed loop is designed with the help of PI controller to achieve the desired set value of $25 \mathrm{~V}$. The charging of on-board SC module and the discharging of fixed SC module is shown in Fig. 4(b).

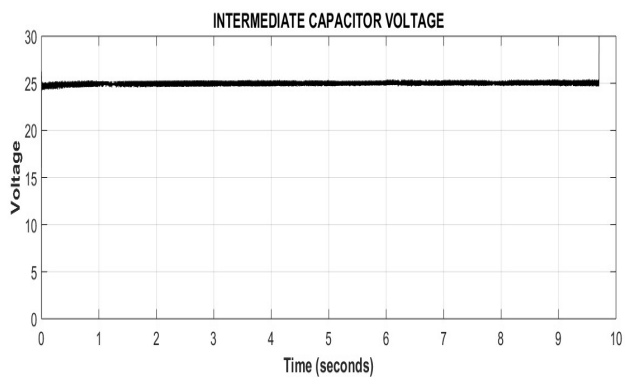

Fig. 4(a): Intermediate Capacitor Voltage

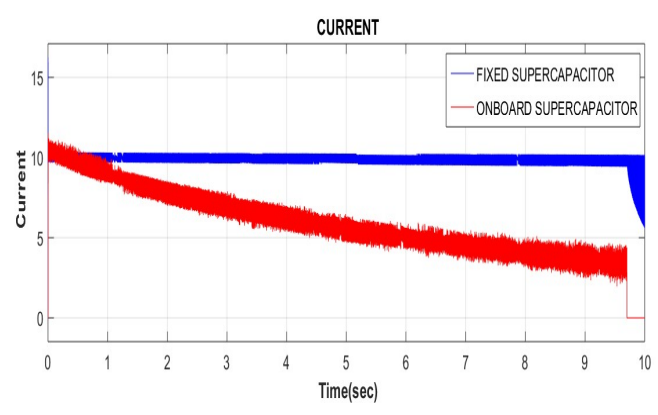

Fig. 4(c): Current waveforms

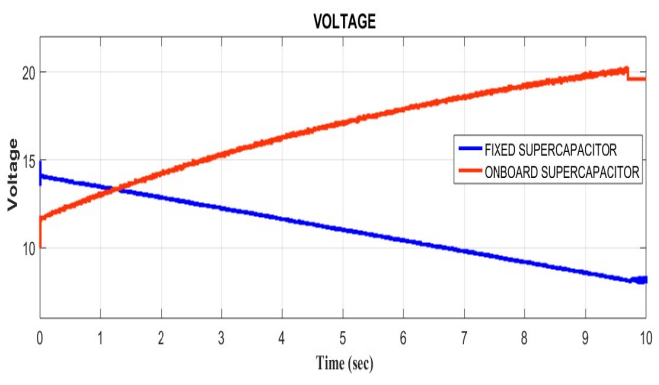

Fig. 4(b): Fixed and On board SC modules

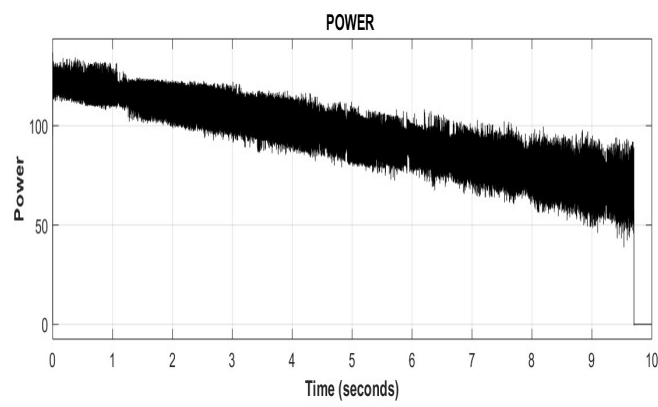

Fig. 4(d): Power Absorbed by On board SC 
It can be observed that the fixed SC module starts discharging from its maximum voltage of $15 \mathrm{~V}$. The on-board $\mathrm{SC}$ module gets charged with respect to time from its initial voltage of $10 \mathrm{~V}$ to its final voltage of $20 \mathrm{~V}$. Fig. 4(c) illustrates the current provided by fixed SC module which is set at a value of $10 \mathrm{~A}$. The current of $10 \mathrm{~A}$ remains constant throughout the energy transfer process which is carried out using PI controller as discussed earlier. The current absorbed by the on-board SC module goes on decreasing with respect to the time. Due to constant current and decreasing voltage of fixed SC module, the power supplied by it also tends to decrease during the energy transfer process. As a result, the power absorbed by on-board SC module shows a falling curve as shown in Fig. 4(d).

\section{Hardware Implementation of Boost Converter}

In hardware implementation, boost converter as well as buck converter is designed separately to design double stage converter. The process consists of calculation of components parameters, selection of suitable control switch and diode based on the system requirements. The boost converter is designed to get nominal output voltage Vout of $25 \mathrm{~V}$ from given input voltage of $15 \mathrm{~V}$. The maximum output current is maintained at 10A.

For calculation of duty cycle, minimum input voltage of $5 \mathrm{~V}$ and $80 \%$ of converter efficiency are considered. The formula for duty cycle is,

$\mathrm{D}=1-\left(\frac{\mathrm{V}_{\text {in }}(\min ) \times \eta}{\mathrm{V}_{\text {out }}}\right), \mathrm{D}=1-\left(\frac{5 \times 0.8}{25}\right)=0.84$, where, $\mathrm{\eta}$ is efficiency of converter

For calculation of inductance, the following formula is used.

$\mathrm{L}=1-\frac{\mathrm{V}_{\text {in }}\left(\mathrm{V}_{\text {out }}-\mathrm{V}_{\text {in }}\right)}{\Delta \mathrm{I}_{\mathrm{L}} \times \mathrm{f}_{\mathrm{s}} \times \mathrm{V}_{\text {out }}}$ where, $\Delta \mathrm{I}_{\mathrm{L}}=(0.2$ to 0.4$) \times \mathrm{I}_{\text {out }}(\max ) \times \frac{\mathrm{V}_{\text {out }}}{\mathrm{V}_{\text {in }}}$

The calculated critical value of inductance for boost converter using above formula is $90 \mu \mathrm{H}$ at $20 \mathrm{kHz}$ switching frequency. For hardware implementation, inductance value is always chosen greater than critical value. To withstand the maximum output voltage of $25 \mathrm{~V}$ and for the maximum output current of 10 amps, MOSFET (IRFZ44N) switch is used. Similarly, diode of MUR1560 is chosen to fulfil the necessary requirements to tolerate the voltage level and current limit.

The prototypes of fixed SC module and boost converter are shown in Fig. 5.1(a) and in Fig. 5.1(b) with all the components mounted and soldered accordingly.

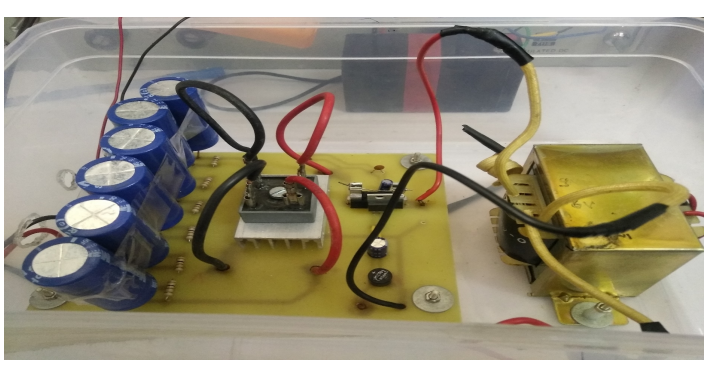

Fig. 5.1(a): Prototype of fixed SC module

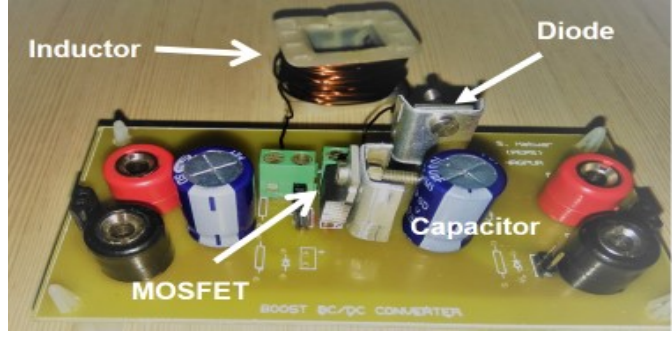

Fig. 5.1(b): Hardware Model of Boost converter

In this paper, closed loop operation for the boost converter is implemented. The Schematic of closed loop boost converter is shown in Fig. 5.2. The drawback of open loop boost converter is decrease of output voltage with respect to time. This drop in voltage is not desired, as it can reduce system efficiency. Therefore, to maintain the output voltage as constant desired value, voltage control loop is designed by providing feedback path which is carried out using a potential divider arrangement. The divider circuit will convert the voltage range of $0-25 \mathrm{~V}$ down to $0-3.3 \mathrm{~V}$ for input to microcontroller. The sense voltage is fed to the ADC module of DsPIC33EP256MC202 microcontroller. The voltage control loop is designed with PI controller code in microcontroller. A set point of $25 \mathrm{~V}$ is considered which must maintain at the output of the converter. The set point value is compared with the sensed voltage to derive an error. The error is processed to the PI control algorithm to decide the pulses which are given to the MOSFET switch. The fixed SC module acts as an input to the converter. This module is charged to $15 \mathrm{~V}$ from single phase AC supply through a rectifier circuit. The switching frequency considered for operation is $20 \mathrm{kHz}$. Fig. 5.3 shows the gate pulses from microcontroller, discharging input voltage of fixed SC module and output voltage of boost converter. It is observed from the Fig. 5.3, the output voltage is maintained constant at $25 \mathrm{~V}$ with boosted value till $35 \%$ discharge of fixed SC module. 


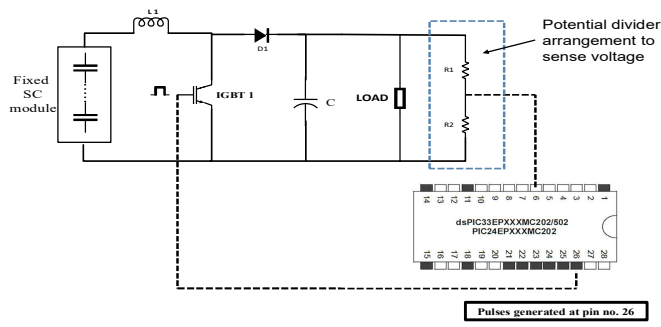

Fig. 5.2: Schematic of Closed Loop Boost converter

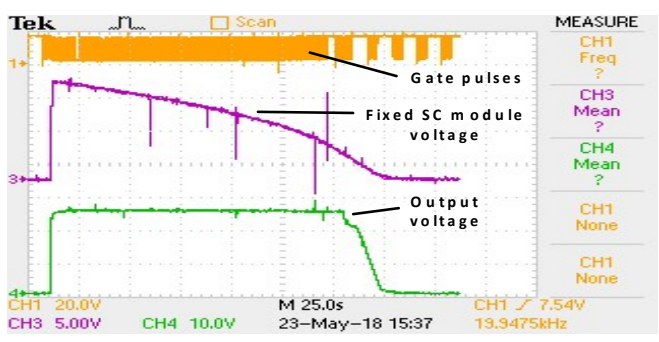

Fig. 5.3: Waveforms of closed loop boost converter

\section{Hardware Implementation of Buck Converter}

The hardware for designing prototype close loop buck converter is implemented separately at continuous current mode (CCM), shown if Fig. 5.4(b) to get nominal output voltage Vout of $20 \mathrm{~V}$ from given input voltage of 25 $\mathrm{V}$. The maximum output current is maintained at 10A. The prototype of on-board SC module, made up of eight 50F, 2.7V supercapacitors, is shown in Fig. 5.4(a).

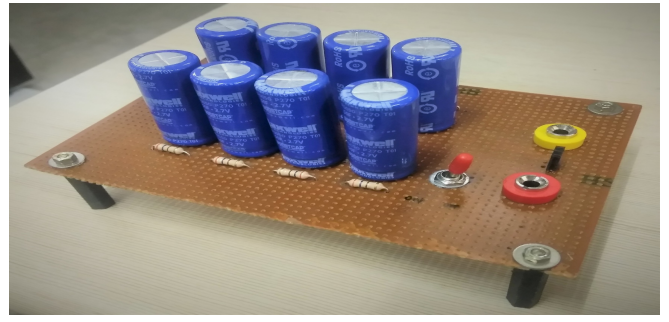

Fig. 5.4(a): Prototype of On-board SC Module

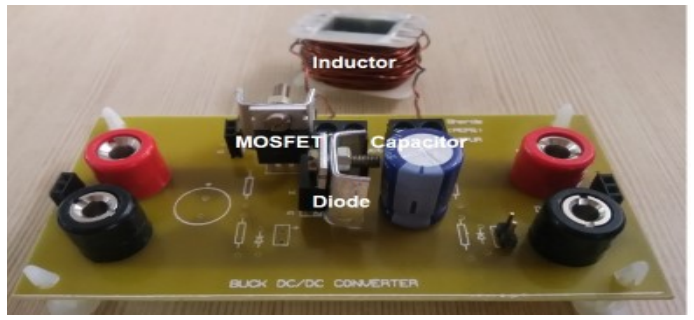

Fig. 5.4(b): Hardware Model of Buck converter

For the given said conditions mentioned above, the inductance value of buck converter is calculated using the same equation as discussed in boost converter at switching frequency of $20 \mathrm{kHz}$. The critical value as per the equation is found out to be $66.66 \mu \mathrm{H}$. For CCM buck converter, value of inductance greater than critical value is chosen. As well as, for selection of power semiconductor devices, MOSFET (IRFZ44N) and Diode (MUR1560) of same ratings are chosen as mention in boost converter designing. The output capacitor $1000 \mu \mathrm{F}, 50 \mathrm{~V}$ is chosen as supporting component.

The closed loop operation is implemented using PI algorithm built in DsPIC33EP256MC202 microcontroller. For testing the validity of the closed loop, the input to the converter is provided by the DC regulated source. The $\mathrm{DC}$ source is initially set at $25 \mathrm{~V}$ which is varied manually to lower the value of voltage. The reference voltage is set at $12 \mathrm{~V}$ which is compared with the actual voltage sensed form the output stage. The input and output waveform can be seen in Fig. 5.5. It can be observed that the output voltage remains constant at desired value of $12 \mathrm{~V}$ even though there is variation in the input voltage.

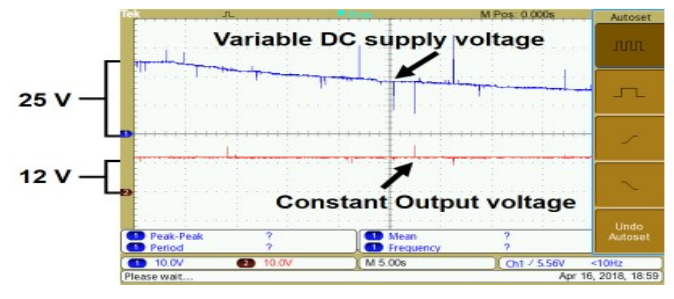

Fig. 5.5: Waveforms of Closed Loop Buck

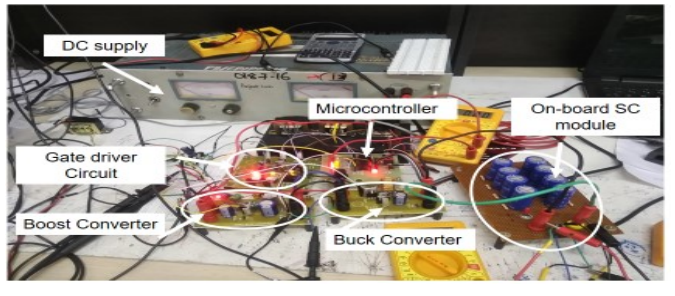

Fig. 5.6: Hardware Layout of Double Stage

\section{Hardware Validation of Double Stage Converter}

The double stage converter is implemented by cascading of boost converter and buck converter. The hardware setup is shown in Fig. 5.6. The double stage converter is tested on regulated DC supply which acts as input to the double stage converter. The control strategy includes current control loop for keeping the current through inductor of first stage as constant. The voltage control loop is employed to maintain the voltage across intermediate capacitor at desired value. The on-board SC module is connected at the output stage of buck converter. 
Fig. 5.7 shows the gate the pulses which are generated by PWM module of DsPIC microcontroller. The yellow colour gate pulses, generated through current control loop, are fed to MOSFET-1 of boost converter circuit. The blue colour gate pulses, generated through voltage control loop, are fed to the MOSFET-2 of buck converter.

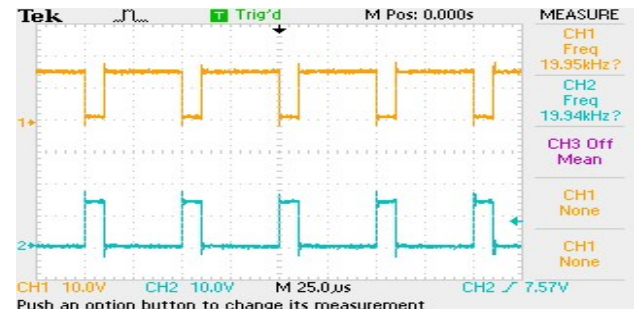

Fig. 5.7: Gate Pulses

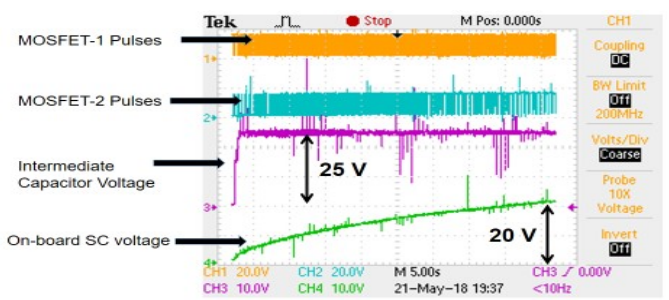

Fig. 5.8: Waveforms of double stage converter

The set point for current control loop is kept at $4 \mathrm{~A}$ while for voltage control loop reference is set at $25 \mathrm{~V}$ during programing the DsPIC microcontroller. The generated waveforms of intermediate capacitor voltage and charged output voltage on on-board SC module are observed as shown in Fig. 5.8. From this figure, it is observed that during experiment, the intermediate capacitor voltage remains constant at $25 \mathrm{~V}$. The on-board SC module gets charged up to a voltage of $20 \mathrm{~V}$ within a time span of approx. $45 \mathrm{sec}$.

\section{Conclusion}

In this paper work, transfer of energy from fixed SC module to on-board SC module has been analysed using simulation and performing experiments. The input current is kept constant while charging on-board SC module. $\mathrm{SC}$ can store/deliver high amount of instantaneous power due to their high power density. This property allows a fast energy exchange between the two SC modules provided that they are interfaced with a suitable convertor. Here, the double stage convertor is used to interface both the SC modules by implementing a proper control strategy. The hardware model of double stage converter is tested on DC regulated supply instead of using fixed SC module as input. The on-board SC module gets charged in $45 \mathrm{sec}$ by controlling current through the DC supply.

\section{References}

[1] R., Rufer and P. Barrade, "A supercapacitor-based energy-storage system for elevators with soft commutated interface", in IEEE Transactions on Industry Applications vol. 38, no. 5, pp. 1151-1159, Sep/Oct 2002. doi: 10.1109/TIA.2002.803021.

[2] B. M. Hasaneen and A. A. Elbaset Mohammed, "Design and simulation of DC/DC boost converter," 2008 12th International Middle-East Power System Conference, Aswan, 2008, pp. 335-340. doi: 10.1109/MEPCON.2008.4562340

[3] S. C. Smith, P. K. Sen and B. Kroposki, "Advancement of energy storage devices and applications in electrical power system," 2008 IEEE Power and Energy Society General Meeting - Conversion and Delivery of Electrical Energy in the 21st Century, Pittsburgh, PA, 2008, pp.1-8.doi: 10.1109/PES.2008.4596436

[4] P. Barrade and A. Rufer, "Power smoothing and high power fast energy exchange between storage systems," in International Conference on Quality and Security of Electrical Supply, (The Oxford Belfry, Thame, U.K.), ERA Technology LTD, 2000.

[5] R. Chakole, M. V. Palandurkar and M. M. Renge, "Energy management of supercapacitor with DC-DC converter," 2016 IEEE 1st International Conference on Power Electronics, Intelligent Control and Energy Systems (ICPEICES), Delhi, 2016, pp.1-6.doi: 10.1109/ICPEICES.2016.7853629

[6] Rufer, P. Barrade, D. Hotellier and S. Hauser, "Sequential supply for electrical transportation vehicles: properties of the fast energy transfer between supercapacitive tanks," 38th IAS Annual Meeting on Conference Record of the Industry Applications Conference, 2003., 2003, pp. 1530-1537 vol.3.doi: 10.1109/IAS.2003.1257759

[7] Youssef Diab, Pascal Venet, Gérard Rojat. Comparison of the Different Circuits Used for Balancing the Voltage of SCs: Studying Performance and Lifetime of SCs. ESSCAP, Nov 2006, Lausanne, Switzerland. pp.on CD, 2006. 